\title{
Effects of Agrobacterial rol-Genes on the Thermodynamic and Structural Features of Starches Extracted from Potato Microtubers
}

\author{
Luybov A. Wasserman ${ }^{1}$, Nina P. Aksenova ${ }^{2}$, Tatiyana N. Konstantinova², Lidiya I. Sergeeva ${ }^{2,3}$, \\ Svetlana A. Golyanovskaya ${ }^{2}$, Alexey V. Krivandin ${ }^{1}$, Georgy A. Romanov ${ }^{2}$ \\ ${ }^{1}$ Emmanuel Institute of Biochemical Physics, Russian Academy of Sciences, Moscow, Russia; ${ }^{2}$ Timiryazev Institute of Plant Physi- \\ ology, Russian Academy of Sciences, Moscow, Russia; ${ }^{3}$ Laboratory of Plant Physiology, Wageningen University, Wageningen, The \\ Netherlands. \\ Email: lwasserma@mail.ru, gar@ippras.ru
}

Received October $16^{\text {th }}, 2013$; revised November $16^{\text {th }}, 2013$; accepted November $23^{\text {rd }}, 2013$

Copyright (C) 2014 Luybov A. Wasserman et al. This is an open access article distributed under the Creative Commons Attribution License, which permits unrestricted use, distribution, and reproduction in any medium, provided the original work is properly cited. In accordance of the Creative Commons Attribution License all Copyrights (C) 2014 are reserved for SCIRP and the owner of the intellectual property Luybov A. Wasserman et al. All Copyright (c) 2014 are guarded by law and by SCIRP as a guardian.

\section{ABSTRACT}

Wild-type potato (Solanum tuberosum L.) plants and their transformants harboring agrobacterial rolB or rolC genes under control of the patatin class I promoter were cultured in vitro. These plants were used as a source of single-node stem cuttings. The structure of native starch in tubers formed on cuttings was determined using methods of X-ray scattering and differential scanning microcalorimetry (DSC). It was found that in starch from tubers of rolB plants the melting temperature of crystalline lamella was lower and their thickness was less than that in wild-type potato. In tubers of rolC plants starch differed from starch in wild-type plants by a higher melting temperature, reduced melting enthalpy, and a greater thickness of crystalline lamellae. The melting of starch from tubers of rolC plants proceeded as the melting of two independent crystalline structures with melting temperatures of $338.0^{\circ} \mathrm{K}$ and $342.8^{\circ} \mathrm{K}$. Overall data show that starches of different structure can be obtained by using transgenic approach.

\section{KEYWORDS}

Solanum tuberosum; Transgenic Potato; Starch; rol Genes; Tubers; Crystalline Lamellae; Melting Temperature

\section{Introduction}

The transition to tuber formation in potato depends on certain environmental and internal cues. External factors, primarily photoperiod, temperature and nutrition supply strongly affect tuber formation [1,2]. Numerous studies showed that phytohormones as internal factors markedly affected all stages of tuberization [3-5]. Agrobacterium rhizogenes rolB and rolC transgenes were used by some researchers to simulate the hormonal control over tuber formation in transformed potato plants [6]. Molecular mode of action of rol transgenes remains debatable; however, it was shown that their effects on higher plants were reminiscent to the effect of phytohormones: auxins in case rolB and cytokinins in case rolC [6]. It was suggested that hormone-like effect of rol genes may be related to their influence on the metabolism of auxins and cytokinins or to changes in cell sensitivity to these phytohormones [7]. It was also shown that the amplitude of the rol gene effect on defined plant organ greatly depended on the used promoters, especially in case of tissue-specific ones [8].

Potato (Solanum tuberosum L.) is an important source of starch, main metabolite of tubers. In spite of numerous investigations, the role of phytohormones in the starch biosynthesis in potato remains unclear. Transgenic plants with modified hormonal status can serve as appropriate model to study this problem. It was shown that rol transgenes induced numerous biochemical and morphological changes, in particular changes in the activity of some 
enzymes of carbohydrate metabolism, the content of soluble sugars [9], and the number and size of starch granules [10]. These changes were somehow similar to those evoked by defined phytohormones [10].

Presently, starch is widely used in different industries, therefore the composition and structural features of this carbohydrate is of great practical importance [11]. The putative influence of rol genes on the qualitative characteristics of storage starch in tuber amyloplasts was so far hardly explored. In this relation, the aim of this work is to study thermodynamic features of starch produced in tubers of potato plants expressing $\mathrm{rolB}$ and $\mathrm{rolC}$ transgenes under control of the tuber-specific patatin class I (B33) promoter.

\section{Materials and Methods}

\subsection{Plant Material}

Investigations were carried out with tubers of wild-type potato (Solanum tuberosum L. cv. Desiree) and transgenic lines of this cultivar expressing rolB and rolC genes of $A$. rhizogenes under the control of the B33 class I patatin promoter. Plants were propagated by in vitro cloning on MS-agar medium containing $60 \mathrm{mg} / \mathrm{l}$ mesoinositol, $0.4 \mathrm{mg} / \mathrm{l}$ thiamine, $0.5 \mathrm{mg} / \mathrm{l}$ pyridoxine, and $2 \%$ sucrose and cultured in a controlled climate chamber at $22^{\circ} \mathrm{C}$ and $16 \mathrm{~h}$ illumination with luminescent lamps producing white light. In the experiments, we used single node stem cuttings with one leaf derived from the middle part of source plants. The cuttings were transplanted to the agar medium of the same composition but supplemented with $8 \%$ sucrose favorable for tuber formation. At 6th day after planting, the cuttings were kept in darkness at $20^{\circ} \mathrm{C}$ $\left(293^{\circ} \mathrm{K}\right)$, which promoted the formation of microtubers. Starch was analyzed in 4 week-old tubers.

\subsection{Starch Content}

Starch was determined in freeze-dried microtubers after prior extraction of soluble sugars with $80 \%$ boiling methanol. Residues were washed with water, lyophilized, repeatedly weighed, and used for hydrolysis of starch as described by Sergeeva et al. [12]. The content of starch was calculated from the level of glucose produced as a result of starch hydrolysis. Glucose was assayed by HPLC combined with pulse amperometric detector using a Dionex system (Dionex, United States) equipped with Carbo PacPA1 columns $(4 \times 250 \mathrm{~mm}$ and $4 \times 500 \mathrm{~mm})$. Elution was conducted with $85 \mathrm{mM} \mathrm{NaOH}$ at room temperature and the flow rate of $1 \mathrm{ml} / \mathrm{min}$.

\subsection{Differential Scanning Microcalorimetry}

Starch was extracted from fresh microtubers at room temperature according to [11] with repeated washing with deionized water. Thermodynamic parameters of melting of $0.15 \%$ water dispersions of investigated starch samples [13] were determined by high-sensitivity differential scanning microcalorimetry (DSC), using a DASM-4 microcalorimeter (Puschino, Moscow Region, Russia). Measurements were conducted in the temperature range from 293 to $373 \mathrm{~K}$ at a constant pressure of $2.5 \mathrm{bar}$ and heating rate of $2 \%$ min. The heat capacity scale was calibrated using the Joule-Lenz effect for each scan. Gelatinization of starch-water dispersions could be considered as quasi-equilibrium process $[14,15]$. It was shown previously that for starches with symmetric DSC-traces the "twostate" model is applicable for the description on the melting process of crystalline lamellae [14,15]. This model implies that melting of starch crystalline lamellae representative the transition of two states, native and molten. Under the chosen experimental conditions, there was no need to take into consideration a thermal lag and duration of the sample treatment in the calorimetric cell [16]. As a reference, deionized water was used. Average values of thermodynamic parameters were calculated on the basis of three measurements and standardized per mole of anhydroglucose (162 g/mol).

The melting temperature corresponded to the maximum on the DSC-thermogram. Repeated scanning showed that the structures melted irreversibly. Melting enthalpy was determined as the area under the peak above the extrapolation curves.

In order to evaluate cooperativity and the thickness of crystalline lamella in starch granules, we used a singlestage model of melting [16]. Values of van't-Hoff enthalpy $\left(\Delta H^{\mathrm{VH}}\right)$ were calculated as described earlier [17] using the following equation

$$
\Delta H^{\nu H}=2 R^{1 / 2} T_{m}\left(C_{p}-0.5 \Delta_{p}^{\mathrm{exp}}\right)^{1 / 2}
$$

where $R$-universal gas constant; $T_{\text {melt }}$-melting temperature of starch crystalline lamella; $C_{p}$-ordinate peak on the DSC-thermogram; $\Delta C_{p}$-difference between heat capacities of melted and native starch dispersions. To calculate the thermodynamic parameters characterising surface of face sides of crystalline lamellae of the starches, symmetrical DSC endotherms were used applying the Thomson-Gibbs' Equation (2) [18,19]:

$$
T_{m}=T_{m}^{o}\left[1-2 \gamma_{i} /\left(\Delta H_{m}^{o} \rho_{c r l} L_{c r l}\right)\right]
$$

where $T_{m}^{o}$ and $\Delta H_{m}^{o}$ are the melting temperature and the melting enthalpy respectively of a hypothetical crystal with unlimited size (a perfect crystal), $\gamma_{i}$ is the free surface energy of face sides of crystalline lamellae, while $\rho_{c r l}$ and $L_{c r l}$ are respectively the density and the thickness of the crystal. Also, the parameter $q_{i}$ which is the surface enthalpy of crystalline lamellae, can be calculated from 
Equations (3)-(4):

$$
q_{i}=\left[\left(\Delta H_{m}^{o}-\Delta H_{\text {exp }}\right) L_{c e l}\right] / 2.5
$$

and

$$
\gamma_{i}=q_{i}-T_{m S i}
$$

Since the values of the melting temperature $\left(T_{m}^{o}\right)$ and the melting enthalpy $\left(\Delta H_{m}^{o}\right.$ ) for a perfect crystal are not available, calculations were performed assuming the values of $T_{m}^{o}(346.8 \mathrm{~K})$ and the $\Delta H_{m}^{o}(35.5 \mathrm{~J} / \mathrm{g})$ for Btype spherulitic crystals [20]. In addition, density, $\rho_{c r l}$, of B-type structures $(1.40 \mathrm{~g} / \mathrm{cm})$ [20] and $L_{c r l}, \Delta H_{m}$ and $T_{m}$ values of the investigated starches were used.

The procedure for deconvolution of melting thermograms of the investigated starch samples has been described previously [14].

\subsection{X-Ray Scattering}

Native starch was investigated by the method of X-ray scattering [21], using an X-ray difractometer equipped with a linear coordinate detector. Debye-Scherrer X-ray optical circuit was employed. X-ray emission was produced by a BSV33Cu sharpfocused X-ray tube with a nickel filter (radiation $\mathrm{CuK} \alpha, \alpha=0.154 \mathrm{~nm}$ ). Before measurement, starch was saturated with distilled water.

\section{Results and Discussion}

Growth characteristics of the tubers of wild type, rolBand rolC plants and total content of starch therein are presented in Table 1, and photos of potato microtubers of different genotypes are shown in Figure 1. Tuber-specific expression of rol transgenes was accompanied by changes in the pattern of tuber formation, including the shape of tubers, their size and number, anatomic structure, phytohormone content and carbohydrate metabolism (sucrose content, activities of invertase, sucrose synthase, fructokinase), that are in correlation with our previously data $[10,22,23]$. As to starch content calculated per g of dry weight, there were no appreciable differences between tubers wild type and rolB plants, whereas in tubers of rolC plants the level of starch was slightly decreased (on the verge of reliability).

DSC-curves and thermodynamic parameters of melting of crystalline lamellae in starch samples are shown in
Figure 2 and Table 2, respectively. We found that melting temperature of starch crystalline lamellae in rolB plants was much lower and in rolC plants much higher than in wild type potato. Enthalpy of starch melting in rolC plants was lower than in rolB and wild type plants. The thickness of crystalline lamella in rolB plants was slightly less and in rolC plants much more than in starch of wild type potato.

The values of the thermodynamic parameters for surface side of the starch lamellae are presented in Table 3. It can be seen that the starch extracted from transgenic rolC microtubers is characterized by an increased value of surface entropy of crystalline lamellae. It is well known that the magnitude of the surface energy is proportional to the amount of defects in the structural organization of starch granules [18,24]. It means that in case of starch extracted from rolC transgenic microtubers we observed an accumulation of defects in crystalline lamellae (i.e. the formation of the energetically unstable state in the starch crystalline region). An accumulation of defects lead to a formation of starch crystals with a more "mellow" surface and to a decrease in their melting temperature.

From DSC-thermograms (Figure 2) it follows that melting curves of starch samples in all the types of plant material display typical for native potato starch thermal transitions which reflect melting of crystalline lamellae of amylopectin [15]. The melting of crystalline lamellae in starch samples isolated from tubers of $r o l B$ and wild type plants were rather symmetric relative to the melting peak. In contrast, in starch of rolC plants, the thermogram of crystalline lamellae melting was asymmetric. Such an asymmetry of the calorimetric melting peak is most likely accounted for by the presence in the starch from rolC tubers of two or more independent ordered structures with different thermostability of crystalline lamellae differing in thickness. Depending on type A or B of polymorphic organization, high-amylose starch can contain from 2 to 3 types of crystalline structures, which melt independently in the same temperature interval [21, 25].

As a rule, the starch from potato tubers belongs to type $\mathrm{B}$; however, our experimental conditions, such as the procedure of transgenic plant production and subsequent long cultivation in vitro, could modify the type of starch polymorphic structure in microtubers. In order to eluci-

Table 1. Growth characteristics of tubers and content of starch therein in wild type and transgenic (rolB and rolC) potato plants after 4 weeks of culturing in continuous darkness.

\begin{tabular}{ccccc}
\hline Plant material & Tuber fresh wt, mg & Length/width ratio of the tuber & Plants with tubers, \% of total number & Content of starch, mg/g dry wt \\
\hline Wild type & $35.1 \pm 3.0$ & $1.20 \pm 0.20$ & $65.5 \pm 7.1$ & $510.5 \pm 25.65$ \\
rolB & $44.5 \pm 2.4$ & $1.09 \pm 0.05$ & $86.2 \pm 4.8$ & $481.5 \pm 15.04$ \\
rolC & $67.2 \pm 5.4$ & $2.71 \pm 0.31$ & $47.0 \pm 4.2$ & $437.8 \pm 14.84$ \\
\hline
\end{tabular}



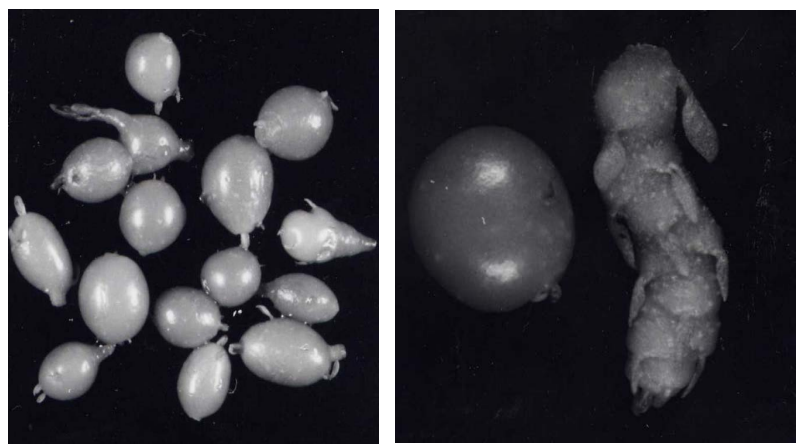

Figure 1. Potato microtubers from wild type (1), transgenic rolB- (2) and rolC (3) plants.

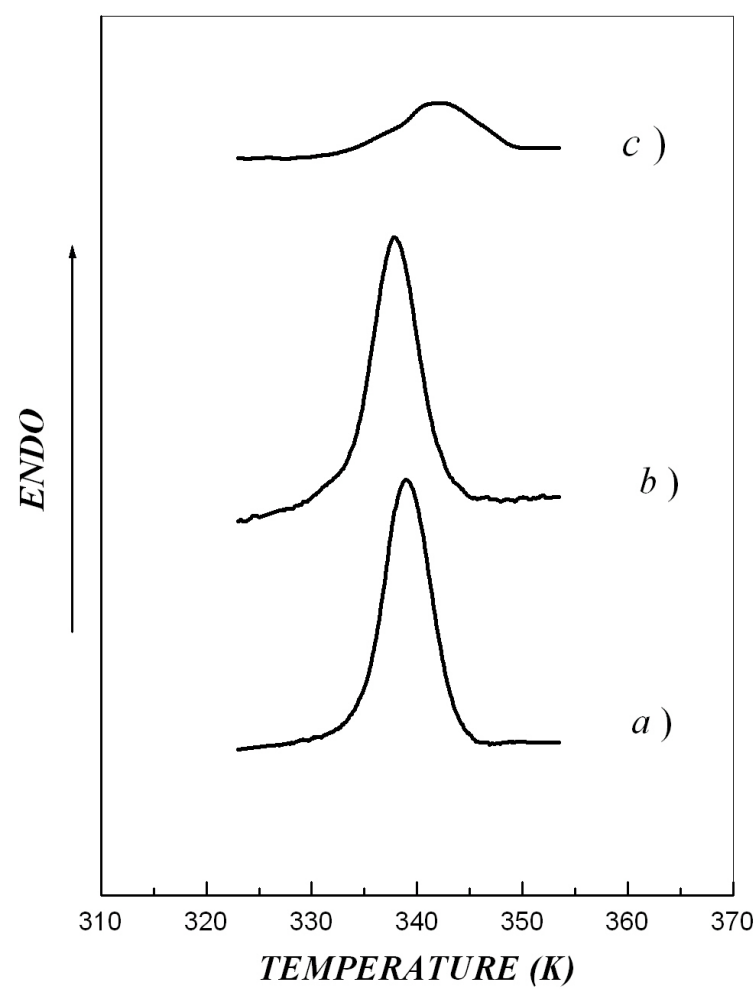

Figure 2. DSC-thermograms for investigated aqueous starch dispersions $\left(\mathrm{C}_{\text {starch }}=0.15 \%\right)$. (a) extracted from microtubers of wild type; (b), transgenic rolB-; (c), transgenic rolC plants).

Table 2. Thermodynamic characteristics of melting (temperature of melting of crystalline lamellae $\left(T_{\text {melt }}\right)$; enthalpy of melting $\left(\Delta H_{\text {melt }}\right)$; Van't Hoff enthalpy $\left(\Delta H^{v H}\right)$; cooperative unit of melting $(v)$; thickness of crystalline lamella $\left(L_{c r l}\right)$ in starches from microtubers of wild type and transgenic (rolB and rolC) potato plants.

\begin{tabular}{cccccc}
\hline $\begin{array}{c}\text { Plant } \\
\text { material }\end{array}$ & $T_{\text {melt }}{ }^{\circ} \mathrm{C}$ & $\begin{array}{c}\Delta H_{\text {melt }}, \\
\mathrm{KJ} / \mathrm{mol}\end{array}$ & $\begin{array}{c}\Delta H^{v H}, \\
\mathrm{KJ} / \mathrm{mol}\end{array}$ & $\begin{array}{c}v \text {, relative } \\
\text { units }\end{array}$ & $L_{\text {crl }}, \mathrm{nm}$ \\
\hline Wild type & $338.9 \pm 0.2$ & $3.6 \pm 0.1$ & $44.9 \pm 0.1$ & $14.5 \pm 0.3$ & $5.1 \pm 0.1$ \\
rolB & $337.9 \pm 0.1$ & $3.2 \pm 0.3$ & $45.5 \pm 0.3$ & $13.5 \pm 0.3$ & $4.7 \pm 0.2$ \\
rolC & $342.1 \pm 0.2$ & $1.0 \pm 0.1$ & $15.9 \pm 0.1$ & $17.9 \pm 0.2$ & $6.3 \pm 0.1$ \\
\hline
\end{tabular}

Table 3. The values of free surface energy $\left(\gamma_{i}\right)$, enthalpy $\left(q_{i}\right)$ and entropy $\left(s_{i}\right)$ of face side for crystalline lamellae of starches extracted from potato microtubers of wild type and transgenic plants.

\begin{tabular}{cccc}
\hline Plant material & $\gamma_{i}^{*} 10^{7} \mathrm{~J} / \mathrm{cm}^{2}$ & $q_{i}^{*} 10^{7} \mathrm{~J} / \mathrm{cm}^{2}$ & $s_{i}^{*} 10^{7} \mathrm{~J} / \mathrm{cm}^{2}$ \\
\hline Wild type & 2.9 & 46.5 & 0.13 \\
rol B & 3.1 & 38.6 & 0.11 \\
rol C & 2.3 & 105.4 & 0.30 \\
\hline
\end{tabular}

date a possible number of crystalline structures, which could cause asymmetry of DSC thermogram describing rolC starch melting, we conducted experiments designed to determine the type of polymorphic organization of starch in the investigated material. For this purpose, the melting of starch from wild type and transgenic (rolB) potato in $0.6 \mathrm{M} \mathrm{KCl}$ (Figure 3) was analyzed. It is known that in the presence of $\mathrm{KCl}$, the melting temperature of crystalline lamella is to become higher [15], thus, melting temperature of A-polymorphic structure of starch usually increases by $7^{\circ} \mathrm{C}-12^{\circ} \mathrm{C}$, whereas melting temperature of B-polymorphic structure increases by only $1^{\circ}$ $4^{\circ}$ [26]. Figure 3 shows that, as compared with aqueous suspension, the melting temperature of starch samples in $\mathrm{KCl}$ solution increased by $1.1^{\circ}-1.3^{\circ}$, which is characteristic of starch with polymorphic structure of type B. The obtained calorimetric results were confirmed by determination of the type of starch polymorphic structure using the method of X-ray scattering (Figure 4). The type of crystalline structure was determined by the location of crystalline reflexes, i.e., by the scattering angle corresponding to the greatest rate of X-ray scattering [27]. Diffractograms of X-ray scattering showed that location of the reflexes for the investigated starch samples corresponded to polymorphic structure of type B.

On the basis of obtained results, melting of starch from the tubers of rolC plants shown in Figure 2 may be regarded as independent melting of two different crystalline structures. The asymmetry of calorimetric melting peak is caused by the melting of two independent ordered structures with different thermostability. Such structures can result from the formation of crystallites with different thickness of crystalline lamellae [15,28]. After heating starch-water dispersion to temperature $T^{*}$, cooling down and further reheating the system, only the calorimetric peak corresponding to melting of more thermostable structure should be observed. We carried out this procedure for starches extracted from microtubers of wild type and transgenic rolB potato plants. Figure 5 shows that, in spite of almost symmetric calorimetric peaks for starches extracted from microtubers of these plants, after the first scan, during reheating there appeared indeed calorimetric peaks corresponding to melting of the structures with higher thermostability. Deconvolution of DSC 


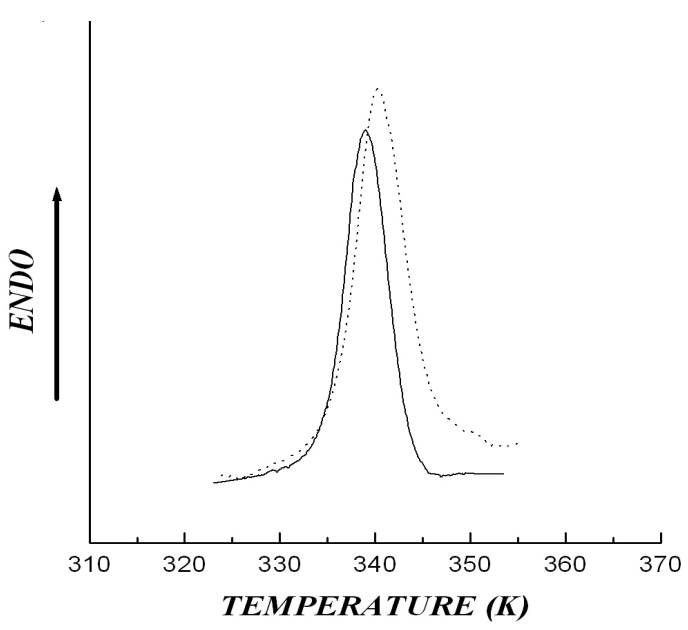

(a)

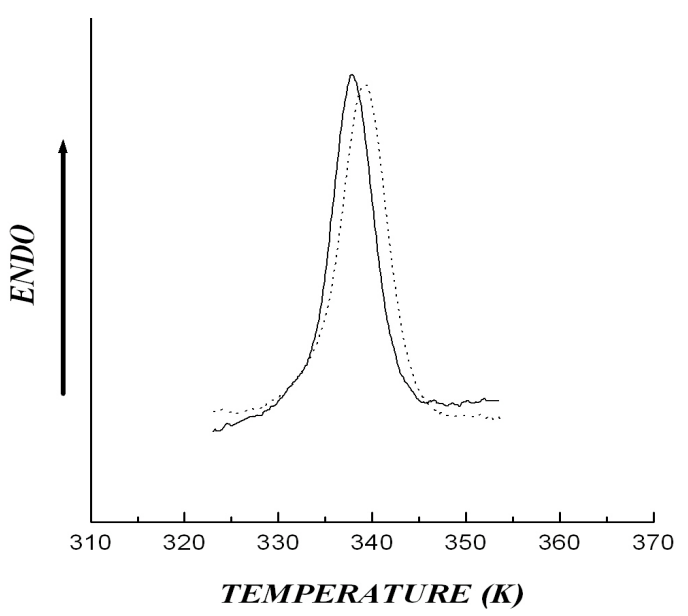

(b)

Figure 3. DSC thermograms for aqueous (solid line) and in 0.6 M KCl (dotted line) for starches extracted from microtubers of wild type (a) and transgenic rolB (b) plants $\left(C_{s t}=\right.$ $0.15 \% \mathrm{w} / \mathrm{w})$.

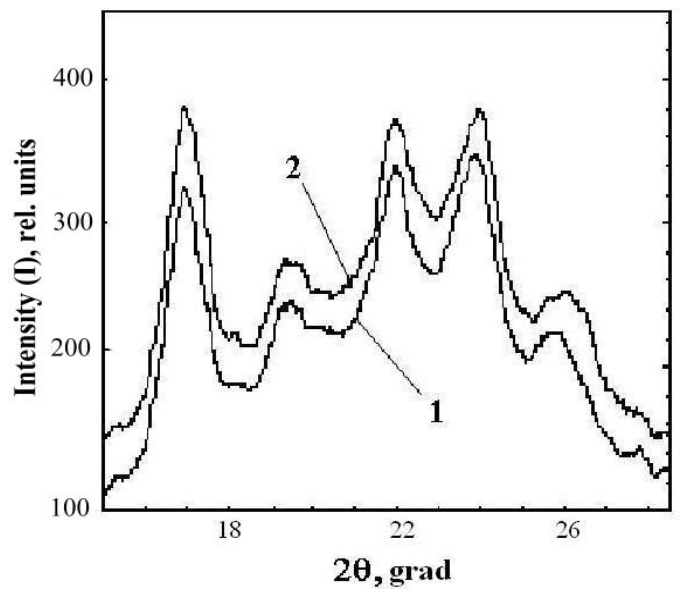

Figure 4. X-ray diffraction pattern for starches extracted from microtubers of wild type (1) and transgenic rolB (2) plants.
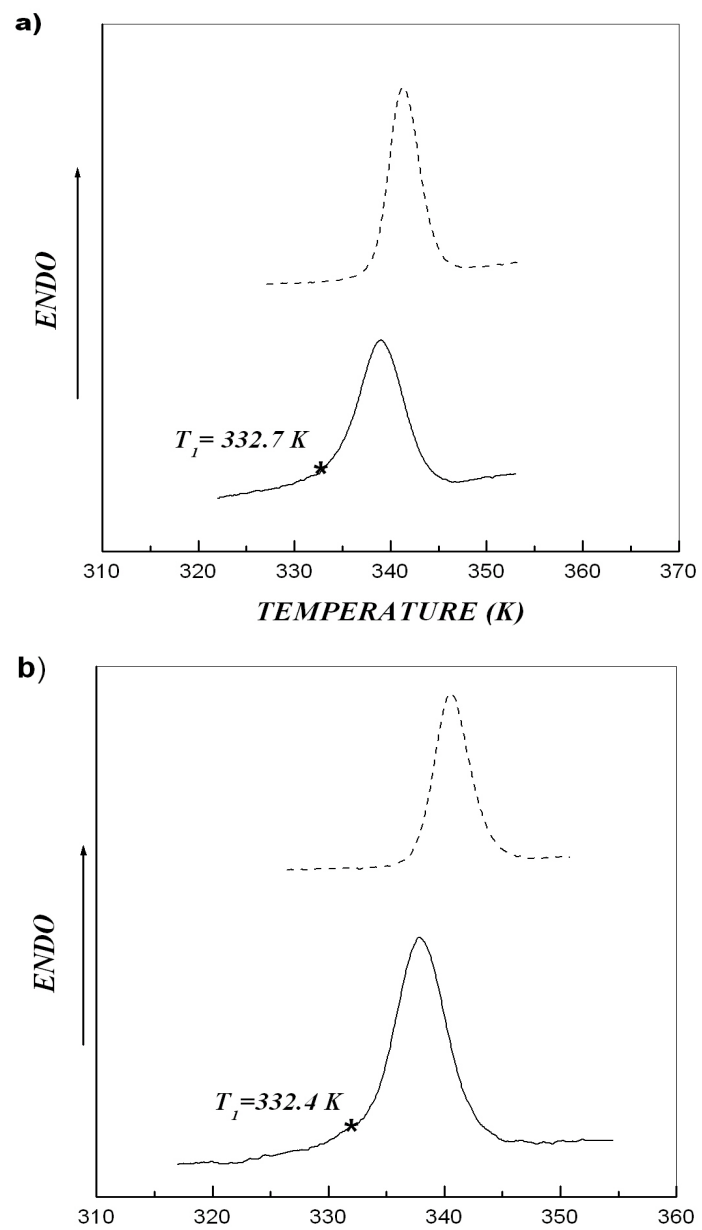

Figure 5. Experimental DSC-curves (solid line) and second heating (dotted line) after annealing for aqueous starch dispersions from potato microtubers of wild type (a) and rolB transgenic (b) plants. Upon reaching the temperature $T_{1}$, the first scan was stopped, the system was cooled down and the second scan (reheating) was made.

thermogram describing melting of starch granules from rolC plants is shown in Figure 6. The thermogram of the melting of starch from rolC tubers may be considered as the curve describing melting of two crystalline structures, with the calculated proportion of low-temperature structure $\left(T_{\text {melt }}=338 \mathrm{~K}\right)$ being $24.4 \%$ and that of high-temperature structure $\left(T_{\text {melt }}=342.8 \mathrm{~K}\right)$ being $75.6 \%$.

According to our experiments, the tuber-specific expression of rol genes considerably affected structural and thermodynamic properties of starch in amyloplasts from tubers of transformed potato. The transgenes rolB and rolC induced somehow opposite changes in the starch crystalline organization. Thus, the melting temperature of crystalline lamellae of starch from the tubers of rolB plants was to some extent lower and in rolC tubers higher than in nontransformed control material. The thickness of starch crystalline lamellae calculated on the basis of 


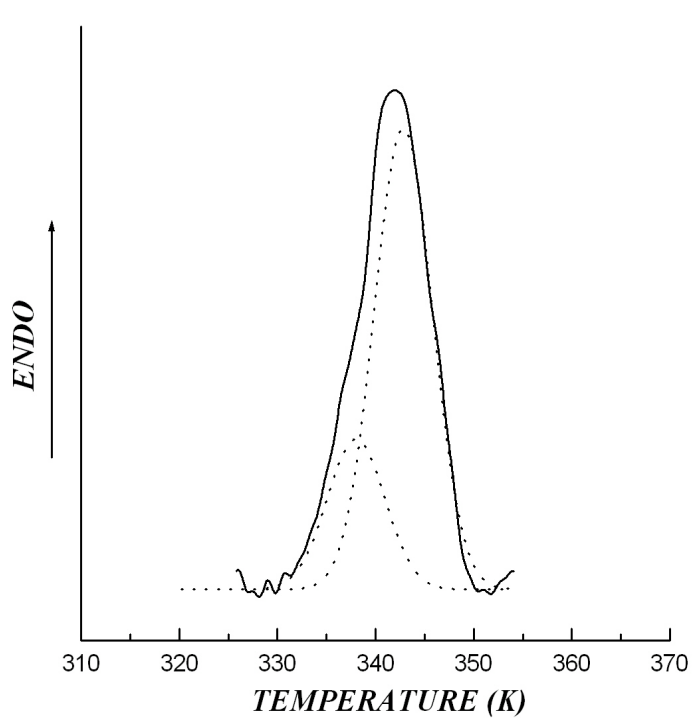

Figure 6. The melting thermograms of starch from transgenic rolC plants of potato after the deconvolution procedure of experimental DSC-traces. The solid line is corresponding to experimental data and dotted lines to calculated data, giving proportion of low-temperature ordered structures with $T_{\text {melt }}=338 \mathrm{~K}$ as $24.4 \%$ and of high-temperature ordered structures with $T_{\text {melt }}=342.8 \mathrm{~K}$ as $75.6 \%$

single stage model of melting was higher in rolC plants and less in rolB transformants compared to wild type plants.

Significant increase in starch melting temperature from rolC tubers evidently depended on numerous disturbances in the structure of crystalline lamellae. As a rule, melting of crystalline lamellae starts with a disintegration of defects in starch crystals, such as chain ends and loop segments [29]. Therefore, elevated temperature of starch melting in rolC tubers suggests that there were the greatest number of defects in this starch, accompanied by a reduction in the value of melting enthalpy and an increase in the thickness of crystalline lamellae. In addition, in contrast to starch from wild type and $\mathrm{rolB}$ plants, melting thermogram of crystalline lamellae in rolC starch was extended and asymmetric (Figure 2), which is characteristic of high-amylose starch [14]. It is possible that the tubers of rolC plants contain storage starch most rich in amylose among all investigated samples. Melting thermograms in $0.6 \mathrm{M} \mathrm{KCl}$ and the results of X-ray scattering confirmed that polymorphic structure of the investigated starch samples belongs to type $\mathrm{B}$. This made it possible to interpret the asymmetry of the melting peak on the DSC-thermogram of starch from the tubers of rolC plants as a result of the presence in this starch of two independent crystalline structures differing in melting temperatures. Deconvolution calculations accomplished for starch of rolC tubers reliably (correlation coefficient $R^{2}=99.4 \%$ ) showed the presence of two dif- ferent crystalline structures. One of them (with melting temperature of $338.0 \mathrm{~K}$ ) accounted for $24.4 \%$ and the other (with melting temperature of $342.8 \mathrm{~K}$ ) for $75.6 \%$.

On the whole, the obtained results revealed a considerable effect of rolB and rolC transgenes on the starch structure in tubers. So far, it is difficult to identify exact pathways of this influence, but one can speculate about possible mechanisms of the effect of investigated transgenes on production and deposition of storage starch.

As it was noted (Figure 2, Table 2), elevated melting temperature of crystalline lamella and low melting enthalpy of starch from rolC plants suggest a high content of imperfect structural areas in this starch. Presently, there accumulate data indicating that one of the functions of starch debranching enzyme (DBE) is a breakdown of wrong structures of starch impeding normal granule growth [10]. Also, the significant increase in average sizes of potato starch granules transformed by BE-I enzyme was observed [30]. It is possible that the accumulation of defects structures in starch from rolC plants is related to the reduced activity of DBE in tubers of these transformants, which could result in the formation of smaller starch granules.

\section{Conclusion}

Our data show that using transgenic approach one can produce starch with different structural characteristics that might have sense for practical use. But further investigations are required to elucidate specific biochemical mechanisms of rolB and rolC transgene effects on the potato starch structure.

\section{Acknowledgements}

We thank Drs L. Willmitzer and J. Kossmann (MaxPlanck Institute of Molecular Plant Physiology, Germany) for providing us with rol-transgenic potato lines. This work was partly supported by the program of the Presidium of the Russian Academy of Sciences "Molecular and Cell Biology”.

\section{REFERENCES}

[1] S. D. Jackson, "Multiple Signaling Pathways Control Tuber Induction in Potato,” Plant Physiology, Vol. 119, No. 1, 1999, pp. 1-8. http://dx.doi.org/10.1104/pp.119.1.1

[2] D. J. Hannapel, "Signalling the Induction of Tuber Formation,” In: D. Vreugdenhil, Ed., Potato Biology and Biotechnology: Advances and Perspectives, Elsevier, Amsterdam, 2007, pp. 237-256. http://dx.doi.org/10.1016/B978-044451018-1/50054-3

[3] E. E. Ewing, "The Role of Hormones in Potato (Solanum tuberosum L.) Tuberization,” In: P. J. Davies, Ed., Plant Hormones. Physiology, Biochemistry and Molecular Bi- 
ology, Kluwer, Dordrecht, 1995, pp. 698-724.

[4] M. Rodríguez-Falcón, J. Bou and S. Prat, "Seasonal Control of Tuberization in Potato: Conserved Elements with the Flowering Response," Annual Review of Plant Biology, Vol. 57, 2006, pp. 151-180.

http://dx.doi.org/10.1146/annurev.arplant.57.032905.1052 24

[5] N. P. Aksenova, T. N. Konstantinova, S. A. Golyanovskaya, L. I. Sergeeva and G. A. Romanov, "Hormonal Regulation of Tuber Formation in Potato Plants," Russian Journal of Plant Physiology, Vol. 59, No. 4, 2012, pp. 451-466. http://dx.doi.org/10.1134/S1021443712040024

[6] G. Ooms, D. Twel, M. E. Bossen, J. H. C. Hoge and M. Burrell, "Developmental Regulation of Ri $\mathrm{T}_{1}$-DNA Gene Expression in Roots, Shoots and Tubers of Transformed Potato (Solanum tuberosum cv Diseree)," Plant Molecular Biology, Vol. 6, No. 5, 1986, pp. 321-330. http://dx.doi.org/10.1007/BF00034939

[7] T. Schmülling, M. Fladung, K. Grossmann and J. Schell, "Hormonal Content and Sensitivity of Transgenic Tobacco and Potato Plants Expressing rol-Genes of Agrobacterium rhizogenes T-DNA,” The Plant Journal, Vol. 3, No. 3, 1993, pp. 371-382.

http://dx.doi.org/10.1046/j.1365-313X.1993.t01-20-0099 $\underline{9 . x}$

[8] M. Fladung, A. Ballvora and T. Schmülling, "Constitutive of Light Regulated Expression of the rolC Gene in Transgenic Potato Plants has Different Effects on Yield Attributes and Tuber Carbohydrate Composition," Plant Molecular Biology, Vol. 23, No. 4, 1993, pp. 749-757. http://dx.doi.org/10.1007/BF00021530

[9] E. V. Grishunina, "Effects of rol-Genes on Carbohydrate Metabolism during Tuberization in Potato Plants,” Ph.D. Thesis, Inst. Plant Physiol. RAS, Moscow, 2006. (in Russian)

[10] I. A. Gukasyan, S. A. Golyanovskaya, E. V. Grishunina, T. N. Konstantinova, N. P. Aksenova and G. A. Romanov, "Effect of rol-Transgenes, IAA, and Kinetin on Starch Content and the Size of Starch Granules in Tubers of in Vitro Potato Plants,” Russian Journal of Plant Physiology, Vol. 52, No. 6, 2005, pp. 809-813. http://dx.doi.org/10.1007/s11183-005-0119-1

[11] J. P. Davis, N. Supatcharee, R. L. Khandelwal and R. N. Chibbar, "Synthesis of Novel Starches in Planta: Opportunities and Challenges," Starch-Starke, Vol. 55, No. 3-4, 2003, pp. 107-120. http://dx.doi.org/10.1002/star.200390036

[12] L. I. Sergeeva, S. M. De Bruijon, E. A. M. Koot-Grosveld, O. Navratil and D. Vreugdenhil, "Tuber Morphology and Starch Accumulation are Independent Phenomena: Evidence from ipt-Transgenic Potato Lines,” Physiologia Plantarum, Vol. 108, No. 4, 2000, pp. 435-443. http://dx.doi.org/10.1034/j.1399-3054.2000.100414.x

[13] M. Richter, S. Augustat and F. Schierbaum, "Ausgewahlte Methoden der Starkechemie,” Wissenschaftlliche Verlagsgesellschaft, GmbH, Stuttgart, 1968.

[14] Y. I. Matveev, J. J. G. van Soest, C. Nieman, L. A. Wasserman, V. A. Protserov, M. G. Ezernitskaja and V. P.
Yuryev, "The Relationship between Thermodynamic and Structural Properties of Low and High Amylose Maize Starches," Carbohydrate Polymers, Vol. 44, No. 2, 2001, pp. 151-160.

http://dx.doi.org/10.1016/S0144-8617(00)00211-3

[15] V. P. Yuryev, L. A. Wasserman, N. R. Andreev and V. B. Tolstoguzov, "Structural and Thermodynamic Features of Low- and High-Amylose Starches. A Review,” In: V. P. Yuryev, A. Cesaro and W. Bergthaller, Eds., Starch and starch Containing Origins-Structure, Properties and New Technologies, Nova Science Publishers, New York, 2002, pp. 23-56.

[16] C. G. Billarderis, C. M. Page, L. Slade and R. R. Sirett, “Thermal Behaviour of Amylose-Lipid Complexes," Carbohydrate Polymers, Vol. 5, No. 5, 1985, pp. 367-371. http://dx.doi.org/10.1016/0144-8617(85)90044-X

[17] P. L. Privalov and S. A. Potekhin, "Scanning Microcalorimetry in Studying Temperature-Induced Changes in Proteins," Methods in Enzymology, Vol. 131, 1986, pp. 451.

[18] V. A. Bershtein and V. M. Egorov, "Differential Scanning Calorimetry of Polymers: Physics, Chemistry, Analysis, Technology,” Ellis Horwood, New York, London, Toronto, Sydney, Singapore, 1994.

[19] I. I. Bocharnikova, L. A. Wasserman, A. V. Krivandin, J. Fornal, W. Błaszczak, V. Ya. Chernykh, A. Schiraldi and V. P. Yuryev, "Structure and Thermodynamic Melting Parameters of Wheat Starches with Different Amylose Content," Journal of Thermal Analysis and Calorimetry, Vol. 74, No. 3, 2003, pp. 681-695. http://dx.doi.org/10.1023/B:JTAN.0000011001.02981.88

[20] M. A. Whittam, T. R. Noel and S. Ring, "Melting and Glass/Rubber Transition of Starch Polysaccharides,” In: E. Dickinson, Ed., Food Polymers, Gels and Colloids, Royal Society of Chemistry, London, 1991, pp. 277-278. http://dx.doi.org/10.1533/9781845698331.277

[21] V. P. Yuryev, A. V. Krivandin, V. I. Kiseleva, L. A. Wasserman, N. K. Genkina, J. Fornal, W. Blaszczak and A. Schiraldi, "Structural Parameters of Amylopectin Clusters and Semi-Crystalline Growth Rings in Wheat Starches with Different Amylose Content," Carbohydrate Research, Vol. 339, No. 16, 2004, pp. 2683-2691. http://dx.doi.org/10.1016/j.carres.2004.09.005

[22] N. P. Aksenova, T. N. Konstantinova, S. A. Golyanovskaya, T. Schmülling, J. Kossmann, L. Willmitzer and G. A. Romanov, "In Vitro Growth and Tuber Formation by Transgenic Potato Plants Harboring rolC or rolB Genes under Control of the Patatin Promoter," Russian Journal of Plant Physiology, Vol. 46, No. 4, 1999, pp. 513-519.

[23] N. P. Aksenova, T. N. Konstantinova, S. A. Golyanovskaya, T. Schmülling, J. Kossmann, L. Willmitzer and G. A. Romanov, "Transformed Potato Plants as a Model for Studying the Hormonal and Carbohydrate Regulation of Tuberization,” Russian Journal of Plant Physiology, Vol. 47, No. 3, 2000, pp. 370-379.

[24] V. A. Protserov, L. A. Wasserman, R. F. Tester, S. J. J. Debon, M. G. Ezernitskaja and V. P. Yuryev, "Thermodynamic and Structural Properties of Starches Extracted 
from Potatoes Grown at Differential Environmental Temperatures," Carbohydrate Polymers, Vol. 49, No. 3, 2002, pp. 271-279.

http://dx.doi.org/10.1016/S0144-8617(01)00332-0

[25] Ch. Gernat, S. Radosta, H. Anger and G. Damaschun, "Crystalline Parts of Three Different Conformations Detected in Native and Enzymatically Degraded Starches," Starch/Starke, Vol. 45, No. 9, 1993, pp. 309-314. http://dx.doi.org/10.1002/star.19930450905

[26] T. Ya. Bogracheva, V. J. Morris, S. G. Ring and C. L. Hedley, "The Granular Structure of C-Type Starch and Its Role in Gelatinization,” Biopolymers, Vol. 45, No. 4, 1998, pp. 323-332.

http://dx.doi.org/10.1002/(SICI)1097-0282(19980405)45: 4<323::AID-BIP6>3.0.CO;2-N

[27] P. Cairs, T. Bogracheva, S. G. Ring, L. L. Hedley and V. J. Morris, "Determination of the Polymorphic Composition of Smooth Pea Starch,” Carbohydrate Polymers, Vol. 31, No. 3-4, 1997, pp. 275-282. http://dx.doi.org/10.1016/S0144-8617(96)00115-4
[28] G. O. Kozhevnikov, V. A. Protserov, N. E. Pavlovskaya, L. V. Golischkin, V. N. Milyaev and V. P. Yuryev, "Changes of Thermodynamic and Structural Properties of Wrinkled Pea Starches (Z-301 and Paramazent varieties) During Biosynthesis,” Starch/Starke, Vol. 53, No. 5, 2001, pp. 201-210.

http://dx.doi.org/10.1002/1521-379X(200105)53:5<201:: AID-STAR201>3.0.CO;2-Q

[29] P. J. Jenkins, R. E. Cameron and A. M. Donald, “A Universal Feature in the Structure of Starch Granules from Different Botanical Sources,” Starch/Starke, Vol. 45, No. 12, 1995, pp. 417-420. http://dx.doi.org/10.1002/star.19930451202

[30] L. J. Sweetlove, B. Müller-Röber, L. Willmitzer and S. A. Hill, “The Contribution of Adenosine 5'-Diphosphoglucose Pyrophosphorylase to the Control of Starch Synthesis in Potato Tubers," Planta, Vol. 209, No. 3, 1999, pp. 330-337.

http://dx.doi.org/10.1007/s004250050640 